\title{
COVID-19 vaccine testing in pregnant females is necessary
}

\author{
Sabra L. Klein, ${ }^{1}$ Patrick S. Creisher, ${ }^{1}$ and Irina Burd ${ }^{2}$ \\ 'W. Harry Feinstone, Department of Molecular Microbiology and Immunology, Johns Hopkins Bloomberg School of Public Health, Baltimore, Maryland, USA. Integrated Research Center for Fetal Medicine, \\ Department of Gynecology and Obstetrics, Johns Hopkins University School of Medicine, Baltimore, Maryland, USA.
}

\section{Pregnancy and COVID-19}

According to the US Centers for Disease Control and Prevention (CDC), onequarter of reproductive-aged women (i.e., 15-49 years of age) hospitalized with coronavirus disease 2019 (COVID-19) from March 1-August 22, 2020, were pregnant, and pregnant women were more likely to require mechanical ventilation than nonpregnant women (1). The CDC also reports that cisgender women infected with severe acute respiratory syndrome coronavirus 2 (SARS-CoV-2) during pregnancy are at higher risk for preterm birth (2). Gestation-associated physical, immunological, and endocrinological changes typically place pregnant women and their fetuses at greater risk for severe complications caused by infectious diseases, which is not unique to SARS-CoV-2 (3). Currently, all studies and references are for cisgender women, because there is a paucity of information on SARS-CoV-2 infection and vaccination during transgender pregnancies.

\section{Vaccination in pregnant women} Vaccines are the best defense against many infectious diseases, including coronavirus disease 2019 (COVID-19), with numerous diverse platforms already authorized for use or in phase III randomized clinical trials (4). The COVID-19 mRNA vaccines (Pfizer-BioNTech's BNT162b2 and Moderna's mRNA-1273) currently used for mass vaccination are not live vaccines, nor do they use an adjuvant. As such, the American College of Obstetricians and Gynecologists (ACOG) and the Society for Maternal-Fetal Medicine (SMFM) recommend that COVID-19 vaccines not be withheld from pregnant or lactating women. To date, however, none of the approved COVID-19 vac- cines has been tested for safety, immunogenicity, reactogenicity, or efficacy in pregnant women or for their effects on fetal programming.

The CDC and the independent Advisory Committee on Immunization Practices (ACIP) position on the PfizerBioNTech and Moderna COVID-19 vaccines is that pregnant women included in the current phase I groups recommended to receive the vaccine (e.g., health care workers) should make a personal decision about receiving the vaccine. The CDC and ACIP indicate that pregnant women should discuss this decision with their health care providers, which is consistent with the recommendations of the ACOG and the SMFM and acknowledges the limited evidence available regarding COVID-19 vaccines during pregnancy $(5,6)$. The director of the Center for Biologics Evaluation and Research (CBER) at the FDA made similar remarks at an online press conference about the emergency use authorization (EUA) for the Pfizer-BioNTech vaccine in December 2020 (7). Other than remarks made at a press conference, the FDA has provided no direct guidance on the use of COVID-19 vaccines in pregnant women. Instead, the FDA mentions pregnant women in the EUA letters and fact sheets for health care providers for the individual vaccines (8-11). For example, the EUA letters for both the PfizerBioNTech and Moderna vaccines include stipulations requiring post-authorization observational studies of the vaccines and mention pregnant women as a population of interest for these studies $(9,11)$. Additionally, the EUA fact sheets for health care providers for both vaccines indicate that there is insufficient data on vaccine

Conflict of interest: The authors have declared that no conflict of interest exists.

risk in pregnancy $(8,10)$. Specific to the Moderna vaccine is the mention of a reproductive toxicity study in female rats, in which vaccine-related adverse effects on female fertility, fetal development, and postnatal development were evaluated, with no adverse events reported. There is also a pregnancy exposure registry to monitor pregnancy outcomes for women administered the Moderna vaccine during pregnancy (8). The FDA recognizes the importance of understanding the potential reproductive consequences of COVID-19 vaccines, as evidenced by the recommendation that developmental and reproductive toxicity studies be conducted with potential vaccine candidates (12). Neither Moderna nor Pfizer had put forth statements on their vaccines and pregnancy; thus, the FDA documents and CDC web page are the only resources for health care providers and their patients to determine whether vaccination during pregnancy is appropriate.

\section{Key gaps in knowledge}

Data from German and US trials of the Pfizer-BioNTech mRNA vaccine (BNT162b2) indicate a broad immune response to the vaccine, with induction of neutralizing antibody responses as well as Th1 $\mathrm{CD}^{+}$cells and expansion of effector memory $\mathrm{CD}^{+}$ $\mathrm{T}$ cells in participants (both men and nonpregnant women) $(13,14)$. Whether this same immunophenotypic response occurs in pregnant women is currently unknown. These data raise concerns, because successful pregnancy outcomes are heavily dependent on heightened Th2 and Treg activity, with reduced Th1 responses (3). Disruption of the balance of $\mathrm{CD}^{+} \mathrm{T}$ cell responses during pregnancy is associated with adverse perinatal outcomes, including fetal loss and preterm birth (15). Furthermore, there are concerns that neonates born to mothers with altered $\mathrm{CD} 4^{+} \mathrm{T}$ cell responses may have long-term sequelae (16). 


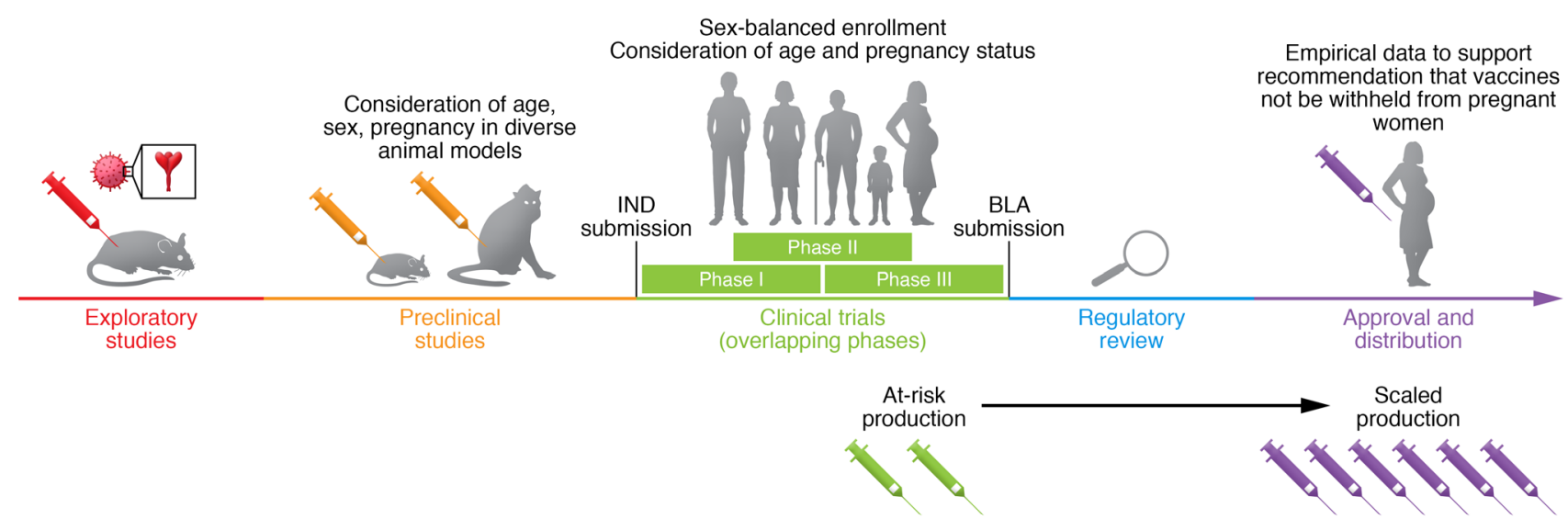

Figure 1. Recommended SARS-CoV2 vaccine development pipeline. The vaccine development pipeline has been accelerated for severe acute respiratory syndrome coronavirus 2 (SARS-CoV-2), including overlapping of clinical trial phases and at-risk production of vaccines still in clinical trials. Despite this, it is vital that factors such as age, sex, and pregnancy be considered throughout the development process. Vaccine platforms are designed in early exploratory studies, and for SARS-CoV-2, much of this discovery was taken from platforms designed for SARS coronavirus (SARS-CoV) and Middle East respiratory syndrome coronavirus (MERS-CoV). These platforms are then tested in preclinical animal models for characterization including safety, toxicity, and immunogenicity. Diverse animal models including rodents and nonhuman primates of both sexes, as well as aged and pregnant animals, can help to elucidate how diverse populations will react to vaccine candidates. After preclinical studies, an investigational new drug (IND) application is submitted, and once approved, the vaccine candidate can be tested in humans in clinical trials. During the enrollment process, there should be sex-balanced enrollment as well as enrollment of older adults and pregnant women if the animal data support safety and efficacy in these groups. With the data from these trials, a biologics license application (BLA) is submitted to regulatory agencies for approval and/or EUA. When SARS-CoV-2 vaccines are widely distributed, it is vital that pregnant women have access to the vaccine and that there are empirical data supporting its safety and efficacy in pregnant women.

The ethics of testing vaccines in pregnant women have been debated for many years (17). Exclusion of women of reproductive age from clinical trials began with the 1974 National Research Act. One goal of this Act was to protect pregnant women and their fetuses from adverse outcomes, but the unintended consequence was the complete exclusion of females despite advocacy for autonomous informed consent (18). In the early 1990s, the FDA and the NIH, with advocacy from US congresswomen, recommended that clinical trials include female subjects (18). Although women are now included in clinical trials of drugs, devices, and biologics, there remains inadequate analysis of whether (a) outcomes differ between females and males, (b) pregnancy alters the effectiveness of treatments, and (c) how treatments affect fetal programming.

None of the COVID-19 vaccine trials has explicitly discussed how risks and other ethical challenges may have impacted trial enrollment of pregnant women, especially when convalescent plasma is safely used in pregnancy (19) and pregnant women are allowed to enroll in the US National Convalescent Plasma Study. No published preclinical animal studies have tested COVID-19 vaccines for safety and toxicity in pregnant females, which is truly a missed opportunity.

Animal models including mice (20), ferrets (21), sheep (22), and nonhuman primates (23) have been used to test other vaccines during pregnancy. The package inserts for Fluzone quadrivalent, FLUAD (i.e., adjuvanted influenza vaccine), BEXSERO (i.e., meningococcal group B vaccine), and SHINGRIX (i.e., adjuvanted, recombinant zoster vaccine) indicate testing in either pregnant rabbits or rats. Although animal models are not a complete representation of human pregnancies (24), they can be used early in the vaccine development pipeline to provide empirical data for inclusion of pregnant women in subsequent clinical trials (Figure 1). Although the FDA recommends that the vaccine pipeline include pregnant animals, there is inadequate oversight to ensure compliance. By partnering with academics in maternal-fetal medicine, immunology, microbiology, and veterinary medicine, preclinical vaccine data could be easily expanded to include data on pregnant females earlier in the vaccine development process (Figure 1).

\section{Conclusions}

It is not ethical to ask pregnant women and their medical providers to make decisions about the COVID-19 vaccine in pregnancy and the implications for the health of the pregnancy and fetal and neonatal development, with little to no empirical evidence upon which to base such decisions. Other than live virus vaccines, there is no ethical reason to not include pregnant women in phase III trials of the COVID-19 vaccines, especially if preclinical safety and toxicology data are available in animal models. Greater consideration of women's health in vaccine studies is encouraged.

Address correspondence to: Sabra L. Klein, Department of Molecular Microbiology and Immunology, Johns Hopkins, Bloomberg School of Public Health, 615, N. Wolfe Street, Baltimore, Maryland 21205, USA. Phone: 410.955.8898; Email: sklein2@jhu.edu.

1. Delahoy MJ, et al. Characteristics and maternal and birth outcomes of hospitalized pregnant women with laboratory-confirmed COVID-19 COVID-NET, 13 states, March 1-August 22, 2020. MMWR Morb Mortal Wkly Rep. 2020;69(38):1347-1354.

2. Woodworth KR, et al. Birth and infant outcomes following laboratory-confirmed SARS-CoV-2 infection in pregnancy - SET-NET, 16 jurisdictions, March 29-October 14, 2020. MMWR Morb Mortal Wkly Rep. 2020;69(44):1635-1640.

3. Vermillion MS, Klein SL. Pregnancy and infection: using disease pathogenesis to inform vac- 
cine strategy. NPJ Vaccines. 2018;3:6.

4. Krammer F. SARS-CoV-2 vaccines in development. Nature. 2020;586(7830):516-527.

5. Centers for Disease Control and Prevention. COVID-19 Vaccination Considerations for People who are Pregnant or Breastfeeding.https:// www.cdc.gov/coronavirus/2019-ncov/vaccines/ recommendations/pregnancy.html. Accessed January 13, 2021.

6. Centers for Disease Control and Prevention. Interim Clinical Considerations for Use of mRNA COVID-19 Vaccines Currently Authorized in the United States.https://www.cdc.gov/vaccines/ covid-19/info-by-product/clinical-considerations. html\#pregnant. Accessed January 13, 2021.

7. FDA not recommending vaccine for pregnant women right now. NBC Boston. December 12, 2020. Accessed February 8 2021. https://www. nbcwashington.com/news/national-international/fda-not-recommending-vaccine-forpregnant-women-right-now/2506288/.FDA not recommending vaccine for pregnant women right now. 2020.

8. U.S. Food and Drug Administration. Fact sheet for healthcare providers administering vaccine (vaccination providers). https://www.fda.gov/ media/144637/download. Updated Deember 20, 2020. Accessed January 13, 2021.

9. U.S. Food and Drug Administration. Moderna COVID-19 vaccine EUA letter of authorization. https://www.fda.gov/media/144636/download. Accessed January 13, 2021.

10. U.S. Food and Drug Administration. Fact sheet for healthcare providers administering vaccine (vaccination providers). https://www.fda.gov/ media/144413/download. Updated January 6, 2021. Accessed January 13, 2021.

11. U.S. Food and Drug Administration. Pfizer COVID19 vaccine EUA letter of authorization reissued 12-23-20. https://www.fda.gov/media/144412/ download. Accessed January 13, 2021.

12. U.S. Food and Drug Administration. Development and licensure of vaccines to prevent COVID-19: guidance for industry. https://www. fda.gov/media/139638/download. Accessed January 13, 2021.

13. Walsh EE, et al. Safety and immunogenicity of two RNA-based COVID-19 vaccine candidates. N EnglJ Med. 2020;383(25):2439-2450.

14. Sahin U, et al. BNT162b2 induces SARS-CoV-2-neutralising antibodies and $\mathrm{T}$ cells in humans [preprint]. https://doi.org/10.1 101/2020.12.09.20245175. Posted on medRxiv December 11, 2020.

15. Saito S, et al. Th1/Th2/Th17 and regulatory T-cell paradigm in pregnancy. Am J Reprod Immunol. 2010;63(6):601-610.

16. Helmo FR, et al. Intrauterine infection, immune system and premature birth. JMatern Fetal Neonatal Med. 2018;31(9):1227-1233.
17. Beeler JA, et al. A systematic review of ethical issues in vaccine studies involving pregnant women. Hum Vaccin Immunother. 2016;12(8):1952-1959.

18. Parekh A, et al. Adverse effects in women: implications for drug development and regulatory policies. Expert Rev Clin Pharmacol. 2011;4(4):453-466.

19. van Griensven J, et al. Evaluation of convalescent plasma for ebola virus disease in Guinea. $N E n g l$ JMed. 2016;374(1):33-42.

20. Hwang SD, et al. Protection of pregnant mice, fetuses and neonates from lethality of H5N1 influenza viruses by maternal vaccination. Vaccine. 2010;28(17):2957-2964.

21. Sweet C, et al. Role of milk-derived IgG in passive maternal protection of neonatal ferrets against influenza. J Gen Virol. 1987;68(Pt 10):2681-2686.

22. Perez-Sancho M, et al. Evaluation of the immunogenicity and safety of Brucella melitensis B115 vaccination in pregnant sheep. Vaccine. 2014;32(16):1877-1881.

23. Paoletti LC, et al. Maternal antibody transfer in baboons and mice vaccinated with a group $B$ streptococcal polysaccharide conjugate. J Infect Dis. 2000;181(2):653-658.

24. Ander SE, et al. Immune responses at the maternal-fetal interface. Sci Immunol. 2019;4(31):eaat6114. 